\title{
Renewal after overexpectation
}

\author{
ROBERT A. RESCORLA \\ University of Pennsylvania, Philadelphia, Pennsylvania
}

\begin{abstract}
Four magazine approach experiments were performed with rats to explore the decrement produced by reinforcing a compound of two previously conditioned stimuli. Compound presentation produced the usual overexpectation decrement in responding to the individual stimuli. However, testing in an alternative, but similarly treated, context allowed renewal of the initial responding to the individual stimuli. This renewal is similar to that seen after a decrement produced by nonreinforcement. It joins other results in suggesting that the decrement produced in extinction and overexpectation may be due to the same mechanism.
\end{abstract}

Overexpectation is one phenomenon that provides striking evidence for a contribution of an error correction mechanism to Pavlovian conditioning. In a typical overexpectation experiment (e.g., Lattal \& Nakajima, 1998; Rescorla, 1970), two conditioned stimuli, A and B, are separately followed by an unconditioned stimulus (US), and then their $\mathrm{AB}$ compound is followed by that same US. The consequence of that additional compound conditioning is that responding to $\mathrm{A}$ and $\mathrm{B}$ decreases relative both to their previous level and to that of similar stimuli not given compound training. This decrease in responding has been documented in a variety of different conditioning preparations (e.g., Kehoe \& White, 2004; Khallad \& Moore, 1996; McNally, Pigg, \& Weidemann, 2004; Wagner, 1971).

These results are consistent with the view that the effectiveness of a US is modulated by the associative strength of the stimuli that precede it. One interpretation is that conditioned changes depend on the discrepancy between the associative strengths of the stimuli on a trial and that which the US can support. Conditioning on a trial thus depends on an error signal that is calculated on that trial (e.g., Rescorla \& Wagner, 1972). Because the total associative strength of $A$ and $B$ together exceeds that which the US can bestow, the discrepancy induced by reinforcement of the compound is negative, resulting in a decrement of responding to the elements.

This way of viewing overexpectation suggests that the decrement that it produces is akin to the decrement that occurs in extinction. There, too, one can see the decrement in responding as resulting from an error calculation that yields a negative result. The previously conditioned stimulus has a level of positive associative strength that the trial outcome, nonreinforcement, cannot support. On this account, the decrements observed in overexpectation and in extinction are attributable to the same underlying process. That observation is of interest for two reasons. First, it is at odds with a commonly held view of extinction, that it represents learning about the so-called no US (e.g., Bou- ton, 1993; Goddard, 1995; Konorski, 1967). On this view, the absence of the US is itself an event that can enter into associations like other events. However, the decrement seen in overexpectation seems unlikely to be of that sort, because it results from the occurrence of a US. Second, if the decrements from overexpectation and nonreinforcement involve the same mechanism, this suggests that overexpectation could provide an alternative procedure for the study of extinction, one that avoids any disruptive effects of omitting the US.

There is some evidence that is consistent with the possibility that overexpectation and extinction involve the same process. Previous reports from this laboratory have shown that two phenomena characteristic of the decrement produced by extinction also occur for the decrement produced by overexpectation. Rescorla (1999), using a procedure in which the association is indexed by outcome-specific transfer to an instrumental response, reported that stimuli subjected to overexpectation continued to have their full original associations, in the manner that is also observed for extinguished stimuli (e.g., Rescorla, 1996). More recently, Rescorla (2006) reported that stimuli subjected to overexpectation show spontaneous recovery with time in the same manner as that shown by stimuli subjected to extinction. Of course, both of these results suggest that overexpectation, like extinction, does not erase the original learning. In that sense, neither of these results is strictly consistent with many error correction models (e.g., Rescorla \& Wagner, 1972). However, those models may correctly describe extinction and overexpectation as sharing the conditions for decrement and an underlying process, despite incorrectly describing the details of that process.

The present experiments were intended to explore the possibility that stimuli exposed to overexpectation also show a third phenomenon frequently observed for extinguished stimuli: renewal of responding with a contextual change. Bouton and his colleagues have documented with both appetitive and aversive procedures that an extin-

R.A. Rescorla, rescorla@cattell.psych.upenn.edu 
guished stimulus can recover a substantial portion of its response if it is tested in a context other than that in which it was extinguished (e.g., Bouton, 1993; Bouton \& Ricker, 1994). This contextual dependence is apparently larger for the decrement produced by extinction than it is for the increment produced by conditioning. Hence, responding to a conditioned and extinguished stimulus recovers with a shift in the context. The goal of the present experiments was to examine whether or not this greater contextual dependence is also a property of the decrement produced by overexpectation.

In the present experiments, a Pavlovian magazine approach procedure was used with rat subjects. In this procedure, a diffuse auditory or visual stimulus signals the delivery of a pellet US in a food magazine. As a result of conditioning, the animal increasingly explores the magazine during the signal, a behavior that is readily monitored. With this procedure, three different renewal paradigms were investigated using overexpectation as the decremental procedure.

\section{EXPERIMENT 1}

This experiment used what has been called an ABA renewal design, in which original training and testing take place in one context (A) but the decremental treatment occurs in a different context (B). The detailed design for Experiment 1 is sketched in Figure 1. Rats received Pavlovian conditioning of two different auditory stimuli: a noise $(\mathrm{N})$ and a clicker $(\mathrm{K})$. Each stimulus was followed by a food pellet $(+)$ in a particular context, A or B. In addition, a diffuse light $(\mathrm{L})$ was reinforced in both contexts. Then one of those auditory stimuli was presented in compound with the light in the alternative context, and that compound was followed by a pellet. In order to produce similar reinforcement experiences for the two contexts, the light was also reinforced separately the same number of times in the other context. Finally, both auditory stimuli were tested in both contexts. This design gives us the opportunity to observe responding to both the treated and the control auditory stimuli both in their original contexts and in a different, but similarly treated, context that was novel for them. Comparisons between stimuli in their treated contexts allows documentation of overexpectation; comparisons in

\begin{tabular}{c|c|c} 
Condition & Over & Test \\
\hline \multirow{2}{*}{ A: L+, N+ } & $\begin{array}{l}\text { A: LK+ } \\
\text { B: L+ }\end{array}$ & \multirow{2}{*}{ A: N, K } \\
\cline { 2 - 2 } B: L+, K+ & $\begin{array}{l}\text { A: L+ } \\
\text { B: } L N+\end{array}$ & B: N, K
\end{tabular}

Figure 1. Design of Experiment 1. Initial conditioning was given with a noise (N) and clicker (K) in two different contexts, $A$ and $B$, as well as with a light $(L)$ in both contexts. Then either $L N$ or LK was reinforced $(+)$ in the training context and $L$ in the other context. Finally, both $\mathrm{N}$ and $\mathrm{K}$ were tested in both contexts. their original training contexts provides the opportunity to observe renewal. Hence, this design constitutes a withinsubjects version of an ABA renewal paradigm.

\section{Method}

\section{Subjects and Apparatus}

The subjects were 16 male Sprague Dawley rats about 150 days old. They were housed in individual cages and placed on a food deprivation regime that kept them at $80 \%$ of their ad lib body weight for the course of the experiment. They had free access to water in the home cage.

The apparatus consisted of four operant chambers measuring $22.9 \times 20.3 \times 20.3 \mathrm{~cm}$, identical to those used in previous reports (e.g., Colwill \& Rescorla, 1985). The two end walls of each chamber were aluminum; the side walls and ceiling were clear Plexiglas. During magazine training, the floor of the chamber was composed of $0.48-\mathrm{cm}$ stainless steel rods, spaced $1.9 \mathrm{~cm}$ apart, center to center. For the remainder of the experiment, the chambers were divided into two sets, differing in the nature of the flooring and in the presence of different olfactory cues, as used by Gottlieb (2005). One set of floors was clear plastic, with a continuous pattern of diamonds approximately $0.5 \mathrm{~cm}$ on a side, each with a raised edge. Evenly spaced $0.35-\mathrm{cm}$ holes were drilled into the floor. The other set of floor covers was smooth aluminum with a continuous pattern of $3.0-\mathrm{cm}$ squares, arranged in an asterisk pattern. Odors were placed in a small open container directly below the floor under the food magazine. The odor accompanying the plastic floor was $4 \% \mathrm{McCor}-$ mick imitation coconut extract; that accompanying the aluminum floor was $2 \%$ McCormick pure anise extract. Each chamber had a recessed food magazine located behind a $4 \times 4 \mathrm{~cm}$ opening in the center of one end wall. An infrared detector and emitter system was mounted on the side walls of the magazine, $1.5 \mathrm{~cm}$ behind the opening and $1.5 \mathrm{~cm}$ from the floor of the magazine, permitting automatic recording of head movements into the magazine.

Each chamber was enclosed in a sound- and light-resistant shell. Mounted on the inside wall of this shell were two speakers that permitted the presentation of a white noise $(\mathrm{N})$ and a $2 / \sec$ clicker $(\mathrm{K})$, each measuring approximately $76 \mathrm{~dB}$ re $20 \mu \mathrm{N} / \mathrm{m}^{2}$ against a background level of $62 \mathrm{~dB}$. Also mounted on that wall was a $6-\mathrm{W}$ bulb that could be illuminated to provide a light $(\mathrm{L})$ stimulus during the otherwise dark session. Another 6-W light, mounted on the ceiling of the chamber, could be flashed on at a rate of $1 / \mathrm{sec}$ to produce a flashing (F) stimulus. The F stimulus was not used in Experiment 1. A food dispenser containing 45-mg pellets (P. J. Noyes, Formula A) allowed delivery of pellets into the food magazine.

Experimental events were controlled and recorded automatically by relays and microprocessors located in an adjoining room.

\section{Procedure}

Magazine training. On the first day, the animals received a 20-min magazine training session, during which 20 noncontingent deliveries of pellets were given, at time intervals variable around a mean of 1 min. During this session, the floor was composed of stainless steel rods, and no odors were present.

Conditioning. On each of the next 12, days the animals received Pavlovian conditioning. On each day, they received two sessions, spaced approximately $3 \mathrm{~h}$ apart, one in each of the contexts. In one context, they received eight $30-\mathrm{sec}$ presentations each of $\mathrm{L}$ and $\mathrm{N}$, each terminating in a pellet; in the other context, they received eight 30 -sec presentations each of $\mathrm{L}$ and $\mathrm{K}$, each terminating in a pellet. In this and all the subsequent phases of the experiment, trials were presented at a mean intertrial interval of $1.5 \mathrm{~min}$. For half the animals, $\mathrm{N}$ was conditioned in the plastic/coconut context, and $\mathrm{K}$ in the aluminum/anise context; for the other half of the animals, the stimuli were interchanged. The order of presentation of contexts for a given animal was counterbalanced across days.

Overexpectation. On each of the next 3 days, the animals continued to receive two sessions a day, each with 16 trials. During one 
session, the animals received overexpectation treatment in which one of the auditory stimuli was presented in a $30-\mathrm{sec}$ simultaneous compound with the light and reinforced with a pellet. This occurred in the context in which the auditory cue had not received conditioning. During the other session, the animals received 16 reinforced presentations of $\mathrm{L}$ alone. For half the animals, the treated auditory stimulus was N, and for half it was K. Similarly, for an orthogonal half of the animals, overexpectation took place in each of the contexts. The scheduled presentation of each auditory stimulus occurred in the context in which the other auditory stimulus had originally received conditioning.

Test. On the next day, each animal received an additional session of overexpectation and control treatment like those on the previous 3 days, except that only eight trials were given, in sessions only half as long. Then, approximately $3 \mathrm{~h}$ later, all the animals received two brief sessions, one in each context, each of which contained two nonreinforced presentations of $\mathrm{N}$ and $\mathrm{K}$. The order of test contexts was balanced, so that for half of the animals, $\mathrm{N}$ was first tested in its training context and $\mathrm{K}$ in its overexpectation context, whereas for the other half of the animals, $\mathrm{K}$ was first tested in its training context and $\mathrm{N}$ in its overexpectation context.

\section{Results and Discussion}

Initial conditioning proceeded smoothly. On the final day of conditioning, the mean responses per minute were 14.6, 15.7, and 6.4 during the light stimulus, the auditory stimulus, and the 30 -sec prestimulus period, respectively. The slight superiority of responding to the auditory stimuli over that to the light was not reliable. On the first day of overexpectation treatment, the mean responses per minute were 17.1 and 5.2 during the compound and precompound trials and 14.8 and 5.0 during the light-alone and prelight trials. Although there was a small superiority in responding to the compound, in comparison with that to the light alone, the difference was not reliable. This nonreliable superiority is common for overexpectation procedures in which between-session comparisons are made (e.g., Lattal \& Nakajima, 1998; Rescorla, 2006). On the final day of overexpectation training, the comparable numbers were $15.8,6.4,15.8$, and 6.1 , not reliably different from those on the first day.

Figure 2 shows responding on the test sessions, during which both the auditory stimulus given overexpectation and the control auditory stimulus were each presented in the training context or in the overexpectation context. Responding is shown separately for each stimulus in each context and for the overall prestimulus responding. The contexts are identified by the decremental treatment procedures, so that the same context is the one in which overexpectation was given and the diff context is the context of training and test. It is clear that responding to the control stimulus was similar whatever the context of testing. The failure of a context shift to affect reliably responding to a simply trained excitor is common in appetitive preparations of this sort (e.g., Bouton \& Peck, 1989). It suggests that with these procedures, simple acquisition, unlike the decrement observed with extinction, is not especially context sensitive.

However, for the treated stimulus, responding was lower in the context in which the animal had experienced overexpectation (same). Responding to the treated stimulus was lower than that to the control stimulus in the overexpecta-

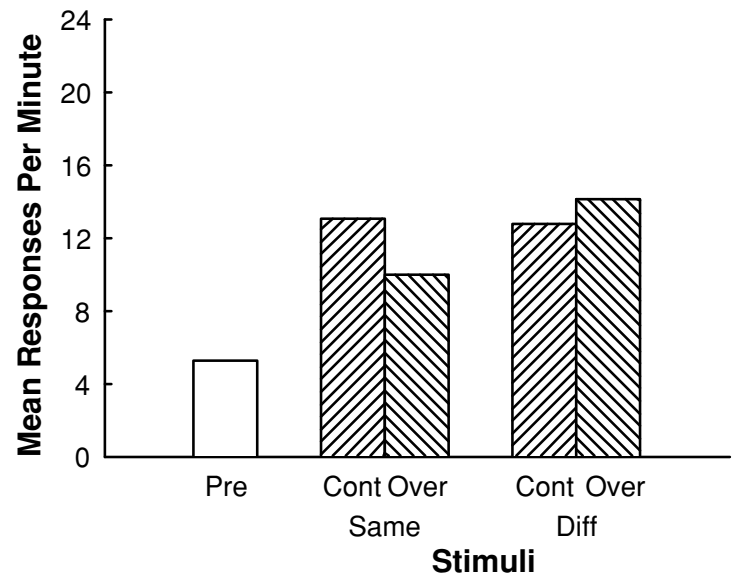

Figure 2. Mean responses per minute during the test in Experiment 1. Responding is shown during the prestimulus (Pre) period, as well as during the control (Cont) stimulus and during the stimulus given overexpectation (Over) both in the same context as that of treatment and in the different context.

tion (same) context [Wilcoxon $T(16)=23.5, p<.05]$. That difference confirms the occurrence of overexpectation. Of more interest, responding to the treated stimulus was lower in the same context in which it had received treatment than was than responding to that stimulus in the different context $[T(13)=13, p<.05]$. That difference suggests a recovery from the decrement induced by overexpectation when the context was shifted.

Consequently, compound presentation reduced responding, but that responding was renewed when the stimulus was tested in the alternative context. These results document the observation of renewal following overexpectation, using an ABA paradigm. As in the case of extinction, the operation of overexpectation produced a decrement that was somewhat specific to the context of the treatment.

\section{EXPERIMENT 2}

The ABA procedure used in Experiment 1 is commonly employed to demonstrate renewal following extinction; however, it is not without its shortcomings. Although there is apparently excellent transfer of responding from the training context to the treatment context, it is not implausible that such a shift would produce underlying generalization decrement. This might result in a relatively easy discrimination between the context in which the stimulus is reinforced and that in which it is extinguished. Consequently, one might expect less than full generalization of the decremental experience back to the training context, producing renewal. This would not require any special sensitivity of the decremental process, relative to the original learning process, to the shift of context.

A somewhat more powerful demonstration of the special contextual dependence of the decremental effects of extinction results from an alternative procedure that might be described as AAB. In this procedure, both the training and the extinction are administered in one context (A), 


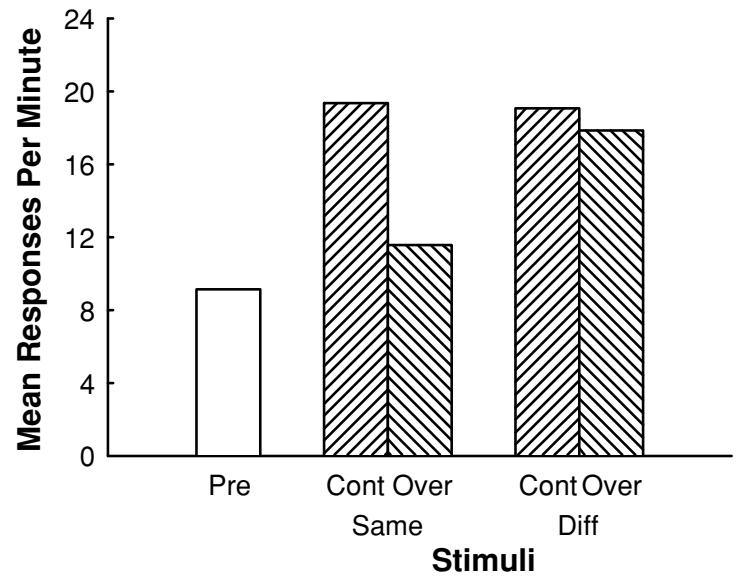

Figure 3. Mean responses per minute during the test in Experiment 2. Responding is shown during the prestimulus (Pre) period, as well as during the control (Cont) stimulus and during the stimulus given overexpectation (Over) both in the same context as that of treatment and in the different context.

and then renewal is tested in a different, but similarly treated, context (B). The observation of renewal under those circumstances suggests that there is greater contextual dependence of the extinction than of the conditioning experience (e.g., Bouton \& Ricker, 1994).

The question in Experiment 2 was whether a similar differential contextual dependence could be observed for the decremental effects of overexpectation with an $\mathrm{AAB}$ design. For this purpose, the procedure in Experiment 2 was identical to that in Experiment 1, except that the context of overexpectation was identical to that of initial conditioning and the context of test was that in which the other auditory stimulus had received its conditioning and overexpectation. The design is the same as that shown in Figure 1, except that the treatments in the A and B contexts were interchanged during the overexpectation phase of the experiment.

\section{Method}

\section{Subjects and Apparatus}

The subjects were 16 male Sprague Dawley rats of the same type and maintained in the same manner as in Experiment 1. The apparatus was the same as that in Experiment 1.

\section{Procedure}

The procedure was identical to that in Experiment 1, except that during the overexpectation phase, the context remained the same for each auditory stimulus as it was in conditioning.

\section{Results and Discussion}

The results of this experiment were highly similar to those of Experiment 1. On the final day of initial conditioning, the mean responses per minute were 14.5, 15.7, and 5.7 during the light stimulus, the auditory stimulus, and the 30 -sec prestimulus period, respectively. On the first day of overexpectation treatment, the mean responses per minute were 15.1 and 5.3 during the compound and precompound trials and 14.3 and 5.1 during the light- alone and prelight trials. On the final day of overexpectation training, the comparable numbers were 15.9, 6.7, 16.3 , and 6.2 , not reliably different from those on the first day.

Figure 3 shows responding in the test sessions, during which both the auditory stimulus given overexpectation and the control auditory stimulus were each presented in the same context as that during training and overexpectation or in the different context. Responding is shown separately for each stimulus in each context and for the overall prestimulus responding. As in Experiment 1, responding to the control stimulus was similar whatever the context of testing. However, for the treated stimulus, responding was substantially lower in the context in which it had experienced overexpectation (labeled "same"). Confirming the occurrence of overexpectation, responding to the treated stimulus was reliably lower than responding to the control stimulus in the treated (same) context $[T(16)=25, p<$ $.05]$. Confirming renewal, responding to the treated stimulus was also lower in the treated context than in the different context $[T(14)=20, p<.05]$. That is, compound presentation reduced responding, but that responding was renewed when the stimulus was tested in the alternative context.

Figures 2 and 3 give the appearance that renewal may have been greater in the AAB paradigm in Experiment 2 than in the ABA paradigm in Experiment 1. This would be somewhat surprising in the light of the generally smaller renewal that is observed in the AAB paradigm when extinction is the decremental procedure. However, it should be noted that the overall rate of test responding was numerically greater in Experiment 2, making both the decrement and its recovery appear larger than in Experiment 1. Overall rates frequently vary from experiment to experiment in our laboratory with the use of this procedure, making between-experiment comparisons risky.

Of most importance, these results confirm the observation of renewal following overexpectation in an $\mathrm{AAB}$ paradigm. Even though renewal involved testing a stimulus in a context in which it had not previously appeared, it engendered recovery from the decremental effects of an overexpectation treatment. The control stimulus did not show a similar pattern of results.

\section{EXPERIMENT 3}

This experiment had two intentions. First, it was a systematic replication of Experiment 2, providing another example of renewal of responding with a context shift following overexpectation training, using an $\mathrm{AAB}$ design. Second, it matched more exactly the experimental histories of the treatment and test contexts. In Experiment 2 , renewal was tested in a context that had received additional reinforced presentations of $\mathrm{L}$ alone, rather than of $\mathrm{L}$ in compound with an auditory exciter. It seems possible that this treatment might have resulted in greater excitatory conditioning in the renewal context than in the treatment context. Since there is evidence that contextual conditioning might summate with conditioning of the explicit stimulus (e.g., Bouton \& Peck, 1989), it might be 
that greater responding in the transfer context represented not a case of renewal but, rather, one of simple summation. Moreover, in Experiments 1 and 2, the separate presentation of $\mathrm{L}+$ in the alternative context can be thought of as generating an $\mathrm{L}+, \mathrm{LN}+$ procedure across contexts, thus deviating from a basic overexpectation procedure.

In order to address these issues, Experiment 3 employed a renewal procedure that treated the contexts identically, but for different stimuli, as illustrated in Figure 4. During initial conditioning, the animals received conditioning of one auditory (either $\mathrm{N}$ or $\mathrm{K}$ ) and one visual stimulus (either $\mathrm{L}$ or $\mathrm{F}$ ) in a particular context and identical conditioning of the alternative auditory and visual stimuli in the other context. Then, during compound treatment, the various auditory and visual stimuli were presented and reinforced in compound in their original contexts. Finally, the auditory stimuli were tested both in their training/overexpectation context and in the context in which the other auditory stimulus had received its training and overexpectation. The issue was whether there would be renewed responding to an auditory stimulus when it was tested in the alternative context, in which a different auditory stimulus had received entirely equivalent treatment. This design, of course, forgoes the control stimuli used in Experiments 1 and 2. But its attraction is that it permits observation of renewal when the general histories of the contexts are the same and, thus, could provide stronger evidence of the context specificity of overexpectation.

\section{Method \\ Subjects and Apparatus \\ The subjects were 16 male Sprague Dawley rats of the same type and maintained in the same manner as in Experiments 1 and 2. The apparatus was the same as that in Experiments 1 and 2.}

\section{Procedure}

Magazine training. Magazine training was conducted in the same manner as in Experiments 1 and 2.

Conditioning. On each of the next 12 days, the animals received Pavlovian conditioning. As in Experiments 1 and 2, on each day, they received two sessions, spaced approximately $3 \mathrm{~h}$ apart, one in each of the contexts. In one context, they received eight 30 -sec presentations of either $\mathrm{L}$ or $\mathrm{F}$ and either $\mathrm{N}$ or $\mathrm{K}$, each terminating in a pellet; in the other context, they received eight 30 -sec presentations of the alternative auditory and visual stimuli, both reinforced. In this and all the subsequent phases of the experiment, trials were presented at a mean intertrial interval of $1.5 \mathrm{~min}$. For half of the animals, $\mathrm{N}$ was conditioned in the plastic/coconut context, and $\mathrm{K}$ in the aluminum/ anise context; for the other half of the animals, the stimuli were interchanged. Within each of these subgroups, half of the animals received $\mathrm{L}$ in the plastic/coconut context and $\mathrm{F}$ in the aluminum/anise context; for the other half, the stimuli were interchanged. The order of presentation of contexts for a given animal was counterbalanced across days.

Overexpectation. On each of the next 3 days, the animals continued to receive two sessions a day, each containing 16 trials. During one session, the animals received overexpectation treatment in which the auditory and visual stimuli previously trained in that context were presented and reinforced in compound. During the other session, the animals received 16 reinforced presentations of alternative auditory and visual stimuli in compound in their original training context.

Test. On the next day, each animal received an additional two sessions of overexpectation treatment, except that only eight trials were

\begin{tabular}{c|c|c} 
Condition & Over & Test \\
\hline A: L+, N+ & A: LN+ & \\
B: $F+, K+$ & B: FK+ & A: N, K \\
\hline A: $L^{+}, K^{+}$ & A: LK+ & B: N, K \\
B: $F+, N+$ & B: FN+ &
\end{tabular}

Figure 4. Design of Experiment 3. Initial conditioning was given with one of two visual ( $L$ and $F$ ) and one of two auditory ( $N$ and $K$ ) stimuli in each of two contexts ( $A$ and $B)$. Then a compound of an auditory and a visual stimulus was reinforced $(+)$ in each context. Finally, both auditory stimuli were tested in both contexts.

given in each session. Then, approximately $3 \mathrm{~h}$ later, all the animals received two brief sessions, one in each context, each of which contained two nonreinforced presentations of $\mathrm{N}$ and $\mathrm{K}$. The order of test contexts was balanced, so that for half of the animals, $\mathrm{N}$ was first tested in its original context and $\mathrm{K}$ in its transfer context, whereas for the other half of the animals, $\mathrm{K}$ was first tested in its original context and $\mathrm{N}$ in its transfer context.

\section{Results and Discussion}

As in the previous experiments, initial conditioning was uneventful. On the final day of conditioning, the mean responses per minute were 15.0 and 5.5 during the stimulus and prestimulus periods, respectively. On the initial day of compound treatment, the mean responses per minute were 14.9 and 4.6, for the compound and precompound periods, respectively. The comparable numbers on the final day of compound treatment were 14.1 and 4.2 , respectively. Neither the differences between compound and element training nor those over the course of compound treatment were reliable.

Figure 5 shows responding to the auditory stimuli on the test trials, separated for the training/overexpectation (same) context and the different context. It is clear that although responding was depressed when the stimulus was tested in the context of training and overexpectation, it was renewed when the stimulus was tested in the alternative context. The superior responding in the different context proved highly reliable $[T(12)=7, p<.01]$. Since the alternative context had been a location in which another auditory stimulus had experienced the same overexpectation treatment, this is an especially powerful demonstration of renewal as a result of context change. Apparently, the depressive effects of overexpectation are specific to the context in which it was treated, not to the general treatment of the context with any stimulus.

\section{EXPERIMENT 4}

A third paradigm sometimes used for the study of renewal after extinction is the $\mathrm{ABC}$ paradigm. In this procedure, original training takes place in one context (A); 


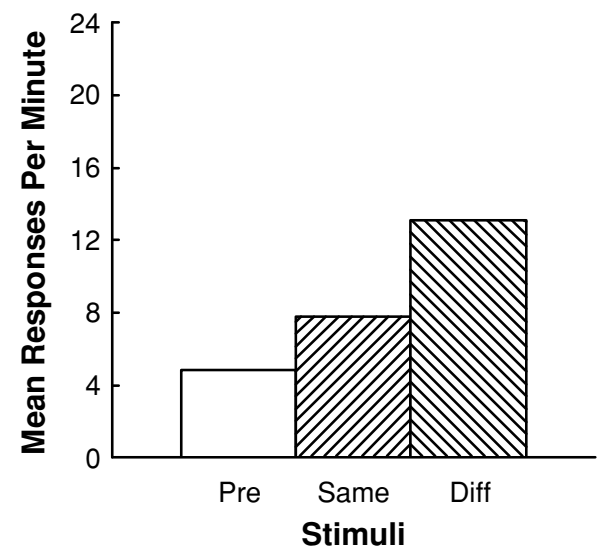

Figure 5. Mean responses per minute during the test in Experiment 3. Responding is shown in the prestimulus (Pre) period, as well as during the stimuli when they were presented either in the same context as that in which they had been subjected to overexpectation or in a different context.

then the decremental procedure takes place in a second context (B), and finally, testing it conducted in yet another context $(\mathrm{C})$. This method shares with the AAB procedure the advantage of studying the impact of a context shift, but it does not require returning the stimulus to a discriminably different context of initial training. Moreover, it avoids giving multiple treatments in the A context, which could make A an ambiguous signal of the conditioning treatment.

A particularly useful application of the $\mathrm{ABC}$ paradigm can be achieved if two different stimuli are initially trained in $\mathrm{A}$ and then one stimulus is extinguished in $\mathrm{B}$, whereas the other is extinguished in $\mathrm{C}$. If both stimuli are tested in both $\mathrm{B}$ and $\mathrm{C}$, the $\mathrm{ABC}$ paradigm can be seen as providing a superior estimate of the renewal observed with a context shift. With this procedure, Contexts $\mathrm{B}$ and $\mathrm{C}$ differ only in that a particular stimulus was extinguished in each. Both the $\mathrm{ABA}$ and the $\mathrm{AAB}$ procedures require comparison of responding in two contexts that differ in both the training and the extinction history for the stimulus of interest. The $\mathrm{ABA}$ procedure compares responding in $\mathrm{A}$, where the stimulus was trained but not extinguished, with that in $\mathrm{B}$, where it was extinguished but not trained. The AAB procedure compares responding in $\mathrm{A}$, where the stimulus was both trained and extinguished, with that in B, where it received neither experience. To the degree that not only extinction but, also, acquisition undergoes some loss with a context shift, the $\mathrm{ABA}$ procedure overstates and the $\mathrm{AAB}$ procedure understates the degree of renewal of extinction (cf. Thomas, Larsen, \& Ayres, 2003). The ABC procedure compares responding in $\mathrm{B}$ and $\mathrm{C}$, neither of which was the context of acquisition and only one of which was the context in which the stimulus was extinguished.

Therefore, Experiment 4 examined renewal for the case of overexpectation, using this $\mathrm{ABC}$ paradigm. The intention was to continue to explore the degree to which the set of renewal phenomena observed after extinction also occurs after overexpectation. For this purpose, the animals received initial conditioning of $\mathrm{L}, \mathrm{N}$, and $\mathrm{K}$ in the standard unlined chamber (A). They then received overexpectation treatment with both auditory stimuli, each in one of the chambers with liners and odors (B and C). Finally, they were tested for the response to both auditory stimuli in both contexts. The issue was whether testing a stimulus in the context in which it had received overexpectation (B) would reveal a decrement that was attenuated when it was tested in a different, but similarly treated, alternative context (C).

\section{Method \\ Subjects and Apparatus \\ The subjects were 16 male Sprague Dawley rats of the same type and maintained in the same manner as in the previous experiments The apparatus was the same as that in the previous experiments.}

\section{Procedure}

Conditioning. Initial magazine training was given in the manner of previous experiments, in the chambers without special floors or odors. On each of the next 12 days, the animals were also given Pavlovian magazine approach training in those same chambers, using procedures like those in the previous experiments. Each day contained eight $30-\mathrm{sec}$ presentations each of $\mathrm{L}, \mathrm{N}$, and $\mathrm{K}$, with a mean intertrial interval of $1.5 \mathrm{~min}$. Trials were given in random order, with the constraint that no more than two trials of a type could occur consecutively; all the trials terminated in the delivery of a pellet.

Overexpectation. On each of the next 3 days, the animals received two sessions a day, one in each of the differential contexts used in the previous experiments. One context had plastic floors and a coconut odor, whereas the other had metal floors and an anise odor. Each session contained 16 trials. During one session, the animals received overexpectation treatment in which one of the auditory stimuli was presented in a 30 -sec simultaneous compound with the light and reinforced with a pellet. During the other session, the animals received 16 reinforced presentations of the other auditory stimulus in compound with the light and reinforced with a pellet. The assignment of auditory stimuli to context was counterbalanced across animals.

Test. On the next day, each animal received an additional session of overexpectation treatment like those on the previous 3 days, except that only eight trials were given, in sessions only half as long. Then, approximately $3 \mathrm{~h}$ later, all the animals received two brief sessions, one in each overexpectation context, each of which contained two nonreinforced presentations of $\mathrm{N}$ and $\mathrm{K}$. The order of test contexts was balanced, so that for half of the animals, $\mathrm{N}$ was first tested in its transfer context and $\mathrm{K}$ in its overexpectation context, whereas for the other half of the animals, $\mathrm{K}$ was first tested in its transfer context and $\mathrm{N}$ in its overexpectation context.

\section{Results and Discussion}

As in the previous experiments, conditioning proceeded smoothly. By the final day of conditioning, the mean responses per minute were 7.2, 17.2, 18.0, and 17.6 during the prestimulus period, the light, the noise, and the clicker, respectively. During the overexpectation treatment phase, the mean responses per minute were 16.3 and 6.3 during the compound and prestimulus periods, respectively, with no systematic changes across days.

Figure 6 shows the results of the test sessions given after a half-session of overexpectation treatment. It is clear that presentation of the stimulus elevated responding, whatever the context. However, the degree of elevation was greater when the stimulus was presented in the context different 
from that in which it had received overexpectation treatment $[T(16)=24, p<.05]$. Comparison with responding in the original training context suggests that there was less responding in the same context, in which overexpectation training had been given $[T(16)=22, p<.05]$. However, that comparison must be treated with caution, because there was no attempt to counterbalance the identity of the training and the overexpectation contexts. Nevertheless, these results suggest that when overexpectation is used in place of extinction in an $\mathrm{ABC}$ paradigm, it produces response decrement with renewal upon context shift.

\section{GENERAL DISCUSSION}

These results confirm prior findings that reinforcing a compound of two previously conditioned stimuli results in a decrease in responding to those stimuli (e.g., Lattal \& Nakajima, 1998) - that is, overexpectation. Of more interest, they found a partial restoration of that responding when the stimuli were tested in a different context - that is, renewal. This renewal was found in all three of the renewal paradigms commonly used following extinction: $\mathrm{ABA}, \mathrm{AAB}$, and $\mathrm{ABC}$. These experiments suggest that an overexpectation treatment can result in a decrement and renewal similar to the decrement and renewal observed in extinction. They are consistent with the view that overexpectation and extinction share a common basis.

Of course, the conclusion that two phenomena are based on the same underlying process can rarely be made with certainty. But the inference that extinction and overexpectation rely on a common process is supported by various kinds of evidence. At the level of dependent variable outcomes, overexpectation appears to display the signature characteristics of extinction: the production of behavioral decrement, the leaving intact of the original associations (e.g., Rescorla, 1999), the occurrence of spontaneous recovery (e.g., Rescorla, 2006), and the renewal of responding with context shift shown here. In addition,

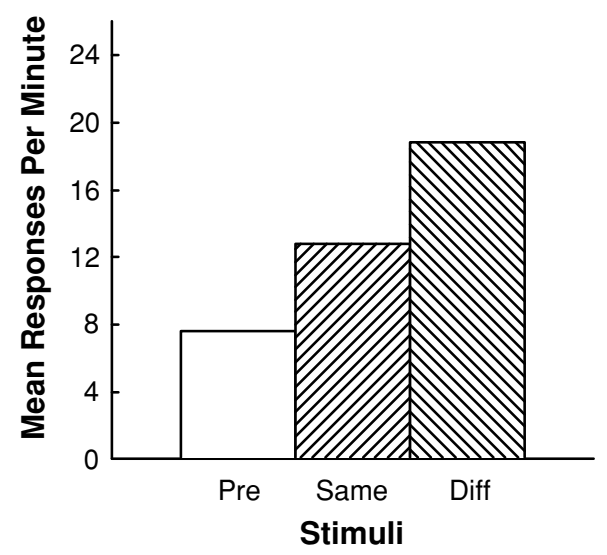

Figure 6. Mean responses per minute during the test in Experiment 4. Responding is shown in the prestimulus (Pre) period, as well as during the stimuli when they were presented either in the same context as that in which they had received overexpectation on in the different context. unpublished experiments in our laboratory have replicated the earlier report of Kremer (1978) that a neutral stimulus present during overexpectation becomes a conditioned inhibitor in the way that a neutral stimulus present during extinction does. Two of these results (spontaneous recovery and renewal) have been found to be relatively specific to extinction, rather than general properties of learning phenomena. Observing those phenomena with overexpectation is, therefore, of particular interest.

There is also evidence that overexpectation and extinction may be sensitive to manipulation of some of the same independent variables. For instance, both greater levels of associative strength and higher stimulus salience result in greater decrements in the case of both extinction (e.g., Rescorla, 2000b) and overexpectation (Kamin \& Gaioni, 1974; Lattal \& Nakajima, 1998). Moreover, McNally et al. (2004) and McNally and Westbrook (2003) have found that in a fear-conditioning preparation, naloxone attenuates the decrement induced by both overexpectation and extinction.

Finally, both overexpectation and extinction seem to be well understood in terms of the same kind of error correction model. Although these observations do not force the conclusion, the similarity of overexpectation and extinction in their characteristics at the levels of the dependent variable, sensitivity to the independent variable, and compatibility with a common theoretical framework strongly encourage the conclusion that they involve a common underlying process.

There are several interesting consequences that follow if one accepts that conclusion. First, it strengthens the claim of error correction models that associative increments and decrements do not depend solely on the absolute value of the trial outcome but, rather, on the relation between the outcome expected and that obtained. Of course, some of those models (e.g., Rescorla \& Wagner, 1972) incorrectly claim that the result of a negative error is the reduction of the original associative learning. Nevertheless, they apparently still correctly characterize overexpectation and nonreinforcement as involving the same mechanism. Second, it weakens the claim that extinction involves the formation of an association between the signal and the no US. Since an easily identified US does occur on overexpectation trials, it seems unlikely that those trials engender an association with a no US. If overexpectation and extinction engage the same process, this makes it less likely that extinction is to be so interpreted, unless the idea of no US receives some additional elaboration.

These results are also consistent with several other procedures that apparently involve decrements while terminating the trial in an effective reinforcer. The first is the replacing of one US by a similarly valued alternative US, after conditioning has been completed. Rescorla (1997) found that such a replacement allowed the full preservation of the associations with the original US, but nevertheless, resulted in the growth of responding with the passage of time, akin to spontaneous recovery. He suggested that using the second US had produced overexpectation that generated an outcome-independent decremental process that dissipated with time. More recently, Rescorla (2000a) 
examined the status of associations over the course of the application of a random procedure in which the US occurred with equal probability in the presence and absence of the signal. He found that although the initial effect of such a treatment was enhanced responding to the signal, resulting from the early establishment of associations with the US, responding declined with continued exposure. However, he also found, using a transfer procedure, that the decline did not result from a loss in the underlying associations. This suggested that the decline in performance resulted from the superimposition of some outcome-independent depressive process. As he noted, if one conceives of both the background and the signal as gaining conditioning during the early application of such a procedure, continued treatment would result in overexpectation during the signal, which would produce the decline in performance but leave the associations intact. Consistent with this analysis, several authors have reported spontaneous recovery after the application of a decremental process involving a random presentation (e.g., Tomie, Hayden, \& Biehl, 1980).

Although the present experiments demonstrate that extinction-like renewal occurs after overexpectation, they offer little insight into the mechanisms of renewal itself. Because, in Experiments 2, 3, and 4, renewal procedures were used that did not involve return to the acquisition context after extinction in a different context (AAB and $A B C$ ), they avoided certain relatively uninteresting possibilities. However, beyond eliminating such alternatives, the present experiments suggest only that, somehow, the mechanism that produces decrement is under the control of the context of that decrement. They do little to specify how that happens. They do not, for instance, allow us to choose between the possibility that the context gains conditional control over the decremental process (e.g., Bouton \& Ricker, 1994) and the possibility that the context and the conditioned stimulus together generate a configural cue that governs a portion of the decremental process. Nor do these results shed light on the nature of the decremental mechanism itself.

Nevertheless, these experiments do show an important commonality between the decrements produced by nonreinforcement and overexpectation. That commonality could have important implications for the description of the conditions producing extinction.

\section{AUTHOR NOTE}

This research was supported by National Institutes of Health Grant R01 MH 67848. Correspondence concerning this article should be addressed to R. A. Rescorla, Department of Psychology, University of Pennsylvania, 3720 Walnut Street, Philadelphia, PA 19104 (e-mail: rescorla@cattell.psych.upenn.edu).

\section{REFERENCES}

Bouton, M. E. (1991). Context and retrieval in extinction and in other examples of interference in simple associative learning. In L. Dachowski \& C. F. Flaherty (Eds.), Current topics in animal learning: Brain, emotion, and cognition (pp. 25-53). Hillsdale, NJ: Erlbaum.

Bouton, M. E. (1993). Context, time, and memory retrieval in the interference paradigms of Pavlovian learning. Psychological Bulletin, 35, 371-390.
Bouton, M. E., \& Peck, C. A. (1989). Context effects on conditioning, extinction, and reinstatement in an appetitive conditioning preparation. Animal Learning \& Behavior, 17, 188-198.

Bouton, M. E., \& RickER, S. T. (1994). Renewal of extinguished responding in a second context. Animal Learning \& Behavior, 22, 317-324.

Colwill, R. M. \& Rescorla, R. A. (1985). Postconditioning devaluation of a reinforcer affects instrumental responding. Journal of Experimental Psychology: Animal Behavior Processes, 11, 120-132.

GODDARD, M. J. (1995). Acquisition of US-no US associations in Pavlovian conditioning. Learning \& Motivation, 26, 264-277.

GotTLieb, D. A. (2005). Acquisition with partial and continuous reinforcement in rat magazine approach. Journal of Experimental Psychology: Animal Behavior Processes, 31,319-333.

KAMIN, L. J., \& GAIONI, S. J. (1974). Compound conditioned emotional response conditioning with differentially salient elements in rats. Journal of Comparative \& Physiological Psychology, 87, 591-597.

KeHOE, J. E., \& White, N. E. (2004). Overexpectation: Response loss during sustained stimulus compounding in the rabbit nictitating membrane preparation. Learning \& Memory, 11, 476-483.

Khallad, Y., \& Moore, J. (1996). Blocking, unblocking, and overexpectation in autoshaping with pigeons. Journal of the Experimental Analysis of Behavior, 65, 575-591.

KONORSKI, J. (1967). Integrative activity of the brain. Chicago: University of Chicago Press.

Kremer, E. F. (1978). The Rescorla-Wagner model: Losses in associative strength in compound conditioned stimuli. Journal of Experimental Psychology: Animal Behavior Processes. 4, 22-36.

LATTAL, K. M., \& NAKAJIMA, S. (1998). Overexpectation in appetitive Pavlovian and instrumental conditioning. Animal Learning \& Behavior. 26, 351-360.

McNally, G. P., Pigg, M., \& Weidemann, G. (2004). Blocking, unblocking, and overexpectation of fear: A role for opioid receptors in the regulation of Pavlovian association formation. Behavioral Neuroscience, 118, 111-120.

MCNALLY, G. P., \& WeSTBRooK, R. F. (2003). Opioid receptors regulate the extinction of Pavlovian fear conditioning. Behavioral Neuroscience, 117, 1292-1301.

RESCORLA, R. A. (1970). Reduction in the effectiveness of reinforcement after prior excitatory conditioning. Learning \& Motivation, 1, 372-381.

REsCORLA, R. A. (1996). Preservation of Pavlovian associations through extinction. Quarterly Journal of Experimental Psychology, 49B, 245-258.

Rescorla, R. A. (1997). Spontaneous recovery after Pavlovian conditioning with multiple outcomes. Animal Learning \& Behavior, 25, 99-107.

RESCORLA, R. A. (1999). Summation and overexpectation with qualitatively different outcomes. Animal Learning \& Behavior, 27, 50-62.

Rescorla, R. A. (2000a). Associative changes with a random CS-US relationship. Quarterly Journal of Experimental Psychology, 53B, 325-340.

RESCORLA, R. A. (2000b). Extinction can be enhanced by a concurrent excitor. Journal of Experimental Psychology: Animal Behavior Processes, 26, 251-260.

RESCORLA, R. A. (2006). Spontaneous recovery from overexpectation. Learning \& Behavior, 34, 13-20.

Rescorla, R. A., \& Wagner, A. R. (1972). A theory of Pavlovian conditioning: Variations in the effectiveness of reinforcement and nonreinforcement. In A. H. Black \& W. F. Prokasy (Eds.), Classical conditioning II: Current research and theory (pp. 64-99). New York: Appleton-Century-Crofts.

Thomas, B. L., Larsen, N., \& Ayres, J. J. B. (2003). Role of context similarity in $\mathrm{ABA}, \mathrm{ABC}$, and $\mathrm{AAB}$ renewal paradigms: Implications for theories of renewal and for treating human phobias. Learning \& Motivation, 34, 410-436.

Tomie, A., Hayden, M., \& BieHL, D. (1980). Effects of response elimination procedures upon the subsequent reacquisition of autoshaping. Animal Learning \& Behavior, 8, 237-244.

WAGNER, A. R. (1971). Elementary associations. In H. H. Kendler \& J. T. Spence (Eds.), Essays in neobehaviorism: A memorial volume to Kenneth W. Spence (pp. 187-213). New York: Appleton-Century-Crofts.

(Manuscript received May 8, 2006; revision accepted for publication August 17, 2006.) 